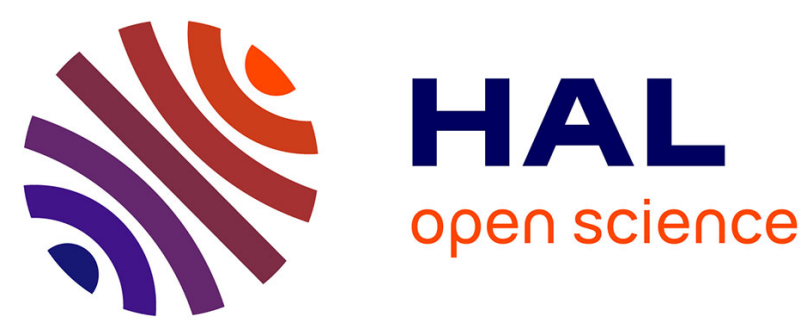

\title{
Plurilinear Modeling and discrete $\mu$-Synthesis Control of a Hysteretic and Creeped Unimorph Piezoelectric Cantilever.
}

\author{
Micky Rakotondrabe, Yassine Haddab, Philippe Lutz
}

\section{- To cite this version:}

Micky Rakotondrabe, Yassine Haddab, Philippe Lutz. Plurilinear Modeling and discrete $\mu$-Synthesis Control of a Hysteretic and Creeped Unimorph Piezoelectric Cantilever.. 9th International Conference on Control, Automation, Robotics and Vision, IEEE ICARV'06., Dec 2006, Singapour, Malaysia. pp.57-64, 10.1109/ICARCV.2006.345129 . hal-00334255

\section{HAL Id: hal-00334255 \\ https://hal.science/hal-00334255}

Submitted on 24 Oct 2008

HAL is a multi-disciplinary open access archive for the deposit and dissemination of scientific research documents, whether they are published or not. The documents may come from teaching and research institutions in France or abroad, or from public or private research centers.
L'archive ouverte pluridisciplinaire HAL, est destinée au dépôt et à la diffusion de documents scientifiques de niveau recherche, publiés ou non, émanant des établissements d'enseignement et de recherche français ou étrangers, des laboratoires publics ou privés. 


\title{
Plurilinear Modeling and discrete $\mu$-Synthesis Control of a Hysteretic and Creeped Unimorph Piezoelectric Cantilever
}

\author{
Micky Rakotondrabe, Yassine Haddab and Philippe Lutz \\ Laboratoire d'Automatique de Besançon - CNRS UMR6596 \\ ENSMM - Université de Franche-Comté \\ 24, rue Alain Savary \\ 25000 Besançon - France \\ \{mrakoton,yhaddab,plutz\}@ens2m.fr
}

\begin{abstract}
First, we present a survey on modeling and control of bending piezoelectric microactuators. Second, a simple model for nonlinear piezoelectric actuators (hysteresis and creep) is presented. It is based on the multilinear approximation. This model requires low computing power and is well adapted for embedded systems. Finally, a $\mu$-synthesis controller is implemented. Experiments show that the obtained performances are compatible with the requirements of micromanipulation tasks.

Index Terms-Piezoelectric cantilever, hysteresis and creep, plurilinear modeling, $\mu$-synthesis control.
\end{abstract}

\section{INTRODUCTION}

Piezoelectric materials have been loyal systems for the actuation of microsystems and microrobots. They offer a good deformation/force ratio as well as a good resolution and rapidity. One of their bountiful applications in the microworld is the actuation of microgrippers, as examples: [1][2][3]. A microgripper can be made of two piezoelectric cantilevers. According to the application, the cantilevers may be controlled on position and/or on force. As part of the modular and re-organizable microfactory project in our laboratory [4][5], each actuator inside the microfactory is considered as a module: it can be removed, added or replaced independantly from the other actuators. On the other hand, to allow the re-organizability of the microfactory, the modularity of the control is also needed. For that, we must consider and study independantly the control of the actuators. A microgripper is composed of two modules: the two cantilevers. The force control and the displacement (bending) control of each cantilever must be studied independently and a high level controller (supervisor) manages the functionning of the whole system. As example, when transporting a micropart, one cantilever is controlled on force while the other on displacement. On the other hand, when picking or releasing a micropart, the two cantilevers are controlled on displacement. However, to complete the modularity of the microfactory, each module should have its own local intelligence. We use a microcontroller for each module where the low level control is implemented.

The aim of this paper is the modeling and the control of the displacement of a piezoelectric cantilever, dedicated to a modular microgripper, subjected to nonlinear phenomena: hysteresis and creeping. Many works have been done for several years about the modeling and control of piezoelectric materials. Thus, the first and second sections are dedicated to a survey on modeling and control of piezoelectric materials. After that, we describe our modeling of the nonlinearity which is based on plurilinear (also called multilinear) approach. Then, the following section presents the design and the results of $\mu$-synthesis controller.

\section{SURVEY ON PIEZOELECTRIC MODELING}

\section{A. Linear modeling}

The static (or quasi-static) linearity phenomenon of a piezoelectric cantilever submitted to mechanical, electrical or thermal excitation has been brought since long time [6]. However, the real physical modeling was first introduced by Smits [7] where, in using strain-energy method, he formulated the slope at the tip $(\alpha)$, the deflexion $\delta$, the displaced volume $v$ and the charge $Q$ versus the mechanical moment $M$, the force $F$, the uniform load $p$ and the voltage $U$ (Fig. 1) for bimorph systems. In [8], he introduced the thermal excitation inside the equations. In the case of a voltage excitation $U$, Rogacheva [9] and Chang [10] shown that the partial slope $\frac{\partial \delta}{\partial U}$ varies according to the applyed frequency. On the other hand, Weinberg extended the physical formulation to multilayered piezoelectric beam [11].

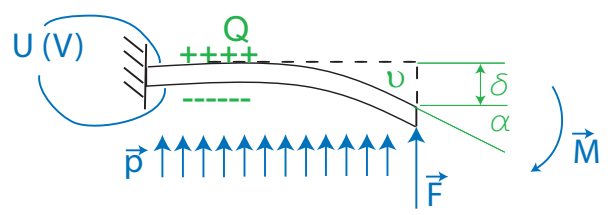

Fig. 1. A piezoelectric cantilever submitted to external excitations. 


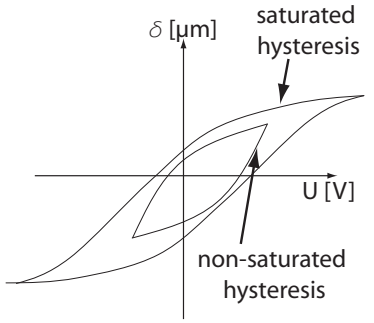

(a)

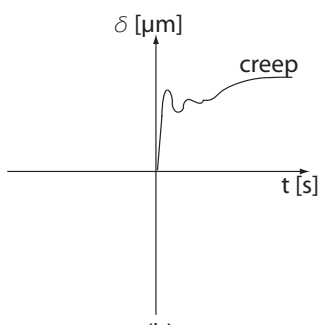

(b)
Fig. 2. a: hysteresis phenomenon. b: creeping phenomenon.

\section{B. Non-linear modeling}

When the deflexion $\delta$ of the beam becomes large, generally higher than $15 \%$ of the maximum field strength [45], the linear modeling is not applicable anymore and hysteresis and creeping phenomena arise (Fig. 2). To model the piezoelectric non-linearity, three categories exist: the microscopic, the semi-macroscopic and the macroscopic modelings.

The microscopic modeling is the less used among the three. It is based on energy relation applied at the atomic or molecular level [12]. This approach presents a high degree of detail but its implementation is very difficult due to the large number of parameters. On the other hand, the semimacroscopic modeling of the piezoelectric materials hysteresis is based both on energy relations for the material and on macroscopic averages to obtain small number of parameters. It combines the Boltzman principles, the domain walls theory and some experiments adjustements (Smith et al [13][14]). Finally, the macroscopic modeling, also called phenomenological modeling, is based on mathematical expressions with parameters determined experimentally. To approach piezoelectric nonlinearity and hysteresis, such works have araised in 90's [15][16][17]. However, the celebrity and arising of mathematical analysis of the Preisach operator have lead the authors to use it since the end of 90's [18][19][20][21][22]. In addition, Lining introduced the notion of tuning voltage to model and to decide about the shape of the hysteresis [23]. The same principle has been done by Low [24]. To complete the phenomenological model of the nonlinear piezoelectric materials, Jung [25] proposed a logarithm based formula to express the time evolution of the creep. In the same aim, Kuhnen [26] introduced the notion of linear creep operator combined with the Preisach operator. The linear creep operator consists on considering the creep as a linear combination of solutions of linear dynamic systems of the first order. In other term, the creep is a generalized Maxwell model. This concept has also been found in [27] coupled with the Preisach operator and in [28] to model the whole hysteresis.

\section{SURVEY ON PIEZOELECTRIC CONTROL}

\section{A. Open-loop control}

The piezoelectric materials, notably beam structures, present nonlinear (hysteresis and creep) characteristics. When applying a known voltage at their input, the repeatability and then the predictability of the output is very bad. Thus, the authors try to linearize the nonlinear behaviour by two methods: model compensation and charge compensation. Both are open-loop control type.

1) Model compensation: An inversion of the hysteresis operator is used and placed before the plant to control. Thus, the whole becomes linear and the quality of the result depends on the exactitude of the hysteresis model and on the inversion method. As examples, [19][22][29] use the Preisach modeling and its inversion for piezoelectric materials. On the other hand, [25][27][30][31] inverse the Preisach model coupled with the creep model. Another way is to consider that the hysteresis is approximated by a phaser with a negative phase for periodic inputs. Cruz-Hernandez introduce the notion of variable phaser to reduce the nonlinearity (hysteresis and saturation) [32][33]. Finally, Lining [23] uses his model with a computer based inversion calculation to linearize the hysteresys of the piezoelectric actuator.

2) Q-charge compensation: While the bending of a piezoelectric beam is nonlinear versus the voltage, it is nearly linear versus the applyed charge. Thus, the authors use that principle in converting the voltage input into a charge input using electronic circuits [34][35]. This method takes into account the hysteresis nonlinearity but not the creep. On the other hand, to obtain a charge control, some authors propose the control of time-integration of a constant current [36][37][38]. According to [39], these methods are not efficient for industrial applications because of the impossibility to maintain the constance of the charge endlessly. Agnus [40] proposes then the $\mathrm{Q} / \mathrm{V}$ control which combines the voltage and the charge controls.

\section{B. Closed-loop control}

When open-loop controlled system is submitted to disturbances, a divergence from the reference point appears and sometimes may generate instability. To solve this problem, closed-loop principle is used. In the case of small displacement where the linearity expression is valid, the PID controller has given good performances. When the displacement is larger, other methods are used to ensure the stability and the performances. Ge [18] and Choi [41] use an inversion principle of the hysteresis model and close the loop with a PID-controller. Other authors [42] [43] propose robust controllers coupled with the model inversion. However, Chen [44] linearizes the hysteresis model of Low [24] before the use of the $H_{\infty}$ based controller. While Zhong [45] uses a semi-macroscopic model coupled with optimal control (minimization of input and output energies) to 
ensure performances, Gorbet employs the Preisach operator and a passivity based controller [46]. Finally, auther authors propose adaptive controllers. As the hysteresis phenomenon is generally non-differentiable, most of them use an artificial intelligency to model in real-time the system and often apply a modulable PID-controller. The real-time modeling or the nonlinear adaptive controller are based either on learning methods [47][48][49] or on neural network principle [50][51][52][53].

\section{Plurilinear modeling}

\section{A. Principle of the static modeling}

The linear modeling is not apropriate when the bending is large enough. Thus, the literature proposes the use of mathematical hysteresis models which are taken into account when synthesising a controller. Sometimes, open-loop control based on non-linearity linearization or compensation is sufficient but when the system is submitted to disturbances, a closed loop controller must be used. Then, many authors propose the use of closed-loop linear controller to the compensed or linearized model. The most of the compensation is based on the Preisach hysteresis model. In this paper, we propose a simple model. As no inversion is used, it does not consume memory and time for the microcontroller. The Fig. 3-a shows the plurilinear approximation of a hysteresis. A variable straightline $(\Delta)$ represents the curve. The offset $\delta_{0}$ and the slope $\alpha$ are dependent on the past and present values of input $U$. We note them $\delta_{U 0}($.$) and \alpha($.$) . Thus, the approximation$ model of the hysteresis without the creep is the pseudolinear formula:

$$
\delta(t)=\alpha(.) \cdot U(t)+\delta_{U 0}(.)
$$

When the external excitations are force $F$ and voltage $U$, the linear formulation of [7] or [11] gives:

$$
\delta(t)=c_{p} \cdot\left(U+\frac{c_{e}}{c_{p}} \cdot F\right)
$$

where $c_{p}$ is the piezoelectric constant and $c_{e}$ the elastic constant.

This expression means that the force behaves like a voltage excitation and vice-versa. Thus, from equ. (1), an external force applyed at the tip of the cantilever would also generate a hysteresis and creeping phenomena. Considering the two excitations, we have:

$$
\delta(t)=\alpha(.) \cdot U(t)+\beta(.) \cdot F(t)+\delta_{U F 0}(.)
$$

Here, $\beta($.$) is the elastic constant depending on the past$ and present value of $F(t)$ and $\delta_{U F 0}($.$) is the new offset$ considering the voltage and the force offsets.

To take into account the creeping, we propose to consider it as an error due to a fictive time-variant force $F_{C}(t)$. The

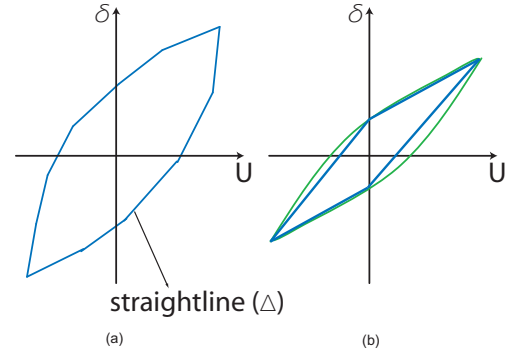

Fig. 3. a: plurilinear approximation of a hysteresis. b: quadrilateral approximation.

final model which includes the hysteretic and creep effects is:

$$
\delta(t)=\alpha(.) \cdot U(t)+\beta(.) \cdot F(t)+\beta_{C}(.) \cdot F_{C}(t)+\delta_{0}(.)
$$

where $\beta_{C}($.$) is the fictive elastic constant and \delta_{0}($.$) an$ equivalent offset.

When the hysteresis does not reach the saturation, a quadrilateral approximation modeling (Fig. 3-b) is sufficient.

\section{B. Quadrilateral modeling in our case}

In our application, the choice of the cantilever is guided by the bending range of the micromanipulation $([-50 \mu m, 50 \mu m])$. To satisfy these constrains, we use a unimorph piezoelectric cantilever based on PIC151 material and a thin $C u$ plate. The total thickness is $0.275 \mathrm{~mm}$ ( 0.2 for the PIC151 and 0.075 for the $\mathrm{Cu}$ ), the width is $2 \mathrm{~mm}$ and the length is $16 \mathrm{~mm}$. These values lead to a non-saturated hysteresis (Fig. 4). The experiments also show the ratedependency of the hysteresis. The non-saturation and the convenable (not very large) area of the hysteresis lead to use the quadrilateral modeling. Let $\left(\Delta_{M}\right)$ and $\left(\Delta_{m}\right)$ represent the two straightlines of the quadrilater with respectively the maximal and the minimal slopes:

$$
\begin{cases}\left(\Delta_{M}\right): & \delta(t)=\alpha_{M} \cdot U(t)+\delta_{M}(.) \\ \left(\Delta_{m}\right): & \delta(t)=\alpha_{m} \cdot U(t)+\delta_{m}(.)\end{cases}
$$

where $\alpha_{M}\left(\alpha_{m}\right)$ represents the maximal (minimal) slope and $\delta_{M}().\left(\delta_{m}().\right)$ represents the general equivalent offset which includes the fictive force, the force applyed to the tip of the cantilever and the equivalent offset as seen in (4).

Let $\alpha_{O}$ be the middle value of the maximal and the minimal slopes and let $\alpha_{E}$ be their radius:

$$
\left\{\begin{array}{l}
\alpha_{O}=\frac{\alpha_{M}+\alpha_{m}}{2} \\
\alpha_{E}=\frac{\alpha_{M}-\alpha_{m}}{2}
\end{array}\right.
$$




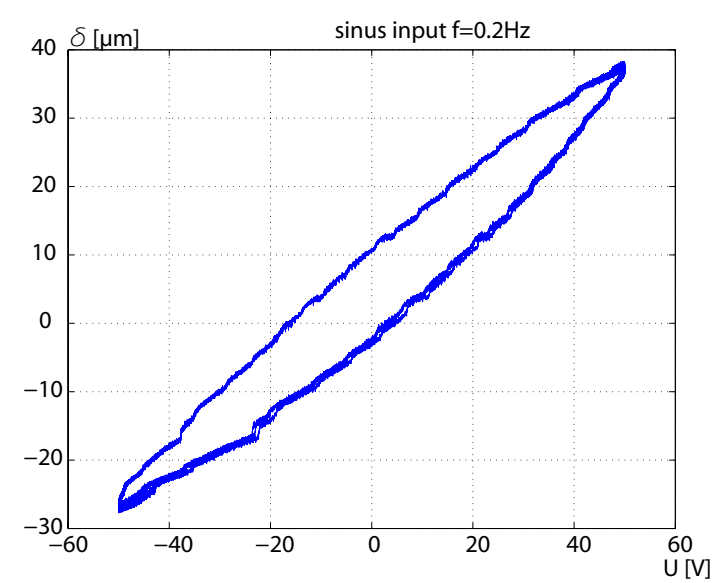

Fig. 4. The unimorph piezoelectric cantilever has non-saturated hysteresis. Here, a sinusoidal voltage with $f=0.2 \mathrm{~Hz}$ and $U=50 \mathrm{~V}$ is applyed.

To model the hysteresis, we propose to use one nominal straightline with an nominal slope and a new general offset $\delta_{P}($.$) :$

$$
\delta(t)=\alpha_{O} \cdot U(t)+\delta_{P}(.)
$$

Thereby, the real system has the following parameters:

$$
\left\{\begin{array}{l}
\delta(t)=\alpha_{\text {real }} \cdot U(t)+\delta_{P}(.) \\
\alpha_{O}-\alpha_{E} \leq \alpha_{\text {real }} \leq \alpha_{O}-\alpha_{E}
\end{array}\right.
$$

Using experimental results, we obtain: $\alpha_{\mathrm{O}}=671 \times 10^{-9}$ and $\alpha_{E}=149 \times 10^{-9}$.

\section{Dynamic identification}

The identification of the dynamic parameters of the cantilever are obtained using the step response (voltage input). We focus on the transient response before the beginning of the creep effect (Fig. 5).

We conclude that a linear $2 n d$ order model is sufficient when the parameters are well adjusted (Fig. 5-dashed plot). Thus, we can complete the model given by the (7) to get the nominal dynamic expression:

$$
\delta(t)=\frac{\alpha_{O} \cdot U(t)+\delta_{P}(.)}{a \cdot p^{2}+b \cdot p+1}
$$

where $\mathrm{a}=6.746 \times 10^{-8}$ is the inertial coefficient and $b=$ $5.195 \times 10^{-6}$ is the viscous coefficient.

Now, the goal is the research of a corrector which stabilizes a nominal model subjected to a disturbance $\frac{\delta_{P}(.)}{\alpha_{O}}$ and where the nominal statical gain $\alpha_{O}$ is followed by an uncertainty (Fig. 6) for the real system.

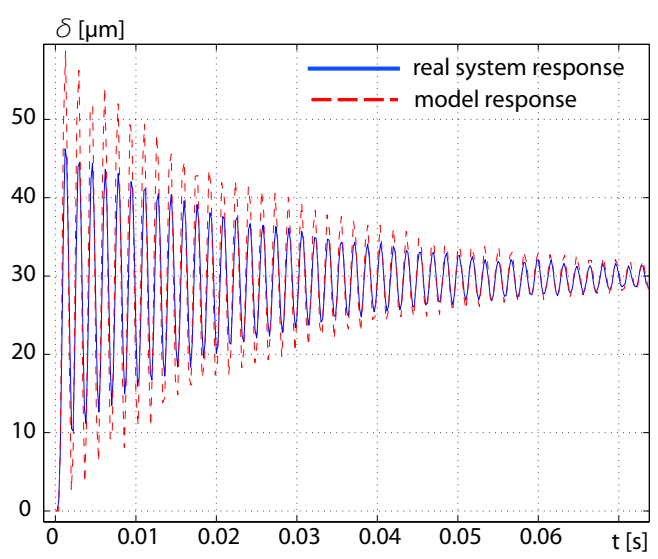

Fig. 5. Response of the piezoelectric cantilever when applying a step voltage signal. Here, we focus on the part before the creep starts in order to identify the dynamic structure and parameters.

\section{DISCRETE $\mu$-SYNTHESIS CONTROL OF THE PIEZOELECTRIC CANTILEVER}

\section{A. discrete $H_{\infty}$ and $\mu$-synthesis}

Let $G(p)$ represent a plant to control where $p$ is the Laplace variable. When synthesising a controller $K(p)$, it is possible to analyze the performances through the bode diagrams characterizing the closed-loop system (Fig. 7-a). If $S(p)=1 /(1+K G)$ is the sensitivity function, these diagrams are given by the following transfer functions:

$$
\left\{\begin{array}{l}
S(j \omega)=\varepsilon / y_{c} \\
K(j \omega) \cdot S(j \omega)=U / y_{c} \\
S(j \omega) \cdot G(j \omega)=y / b \\
K(j \omega) \cdot S(j \omega) \cdot G(j \omega)=y / y_{c}
\end{array}\right.
$$

Where, $\varepsilon$ indicates the error, $y_{c}$ indicates the setpoint, $U$ is the input voltage, $b$ is the external perturbation and $y$ is the output (Fig. 8-a). Thus, to impose some performances, frequential gabarits are used (Fig. 8). These gabarits are dependant on three filters $W_{1}(p), W_{2}(p)$ and $W_{3}(p)$. The standard $H_{\infty}$ problem consists on finding an optimal controller $K$ and an optimal value $\gamma$ so that:

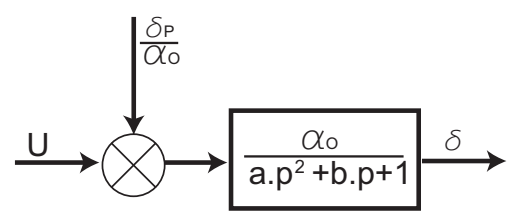

Fig. 6. Scheme of the model to control. 


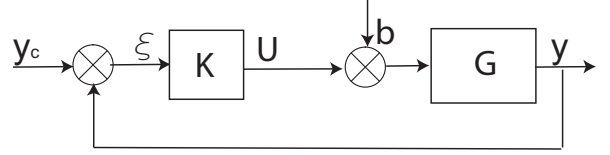

(a)

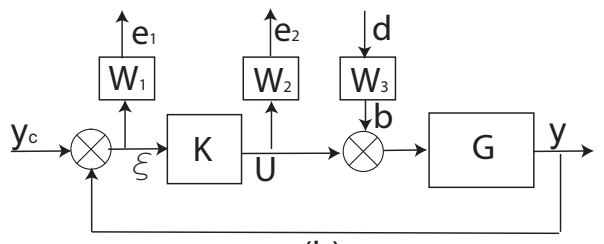

(b)

Fig. 7. a: closed-loop scheme. b: augmented scheme.

$\left\{\begin{array}{l}|S(j w)|<\frac{\gamma}{\left|W_{1}(j w)\right|} \\ |K(j w) S(j w)|<\frac{\gamma}{\left|W_{2}(j w)\right|} \\ |S(j w) G(j w)|<\frac{\gamma}{\left|W_{1}(j w) W_{3}(j w)\right|} \\ |K(j w) S(j w) G(j w)|<\frac{\gamma}{\left|W_{2}(j w) W_{3}(j w)\right|}\end{array}\right.$

which is equvalent to:

$$
\left\{\begin{array}{l}
\left\|W_{1} S\right\|_{\infty}<\gamma \\
\left\|W_{2} K S\right\|_{\infty}<\gamma \\
\left\|W_{1} S G W_{3}\right\|_{\infty}<\gamma \\
\left\|W_{2} K S G W_{3}\right\|_{\infty}<\gamma
\end{array}\right.
$$

Thereby, an augmented closed-loop scheme is obtained (Fig. 7-b). To solve the problem (12), the most used method is the Glover-Doyle algorithm which is based on the Riccati equations [54][55]. The issued controller $K$ is robust in the fact that it ensures the stability and the performances even if the system $G$ has uncertainty vis-à-vis of the real plant. The limit of this uncertainty is found à-posteriori with the $\mu$-analysis tool.

If the uncertainty can be formulated à-priori, the $\mu$ synthesis is the tool which permits finding the optimal controller $K$ and the optimal value $\gamma$ and which ensures the target performances in the uncertainty domain. The $\mu$-synthesis is based on the $H_{\infty}$ and the $\mu$ (structured singular values) tools. It necessitates to structure the uncertainty inside a diagonal matrix $\Delta . \Delta$ is composed of the dynamic uncertainty $\Delta_{i}$, of the parametric uncertainty $\partial_{i}$ and the phase and gain uncertainties $\epsilon_{i}$ :

$\Delta=\operatorname{diag}\left\{\Delta_{1}(s), . ., \Delta_{q}(s), \partial_{1} \cdot I_{r 1}, . ., \partial_{r} I_{r r}, \varepsilon_{1} I_{c 1}, . ., \varepsilon_{c} I_{c c}\right\}$

with: $\Delta_{i}(s) \in R H_{\infty} \quad \partial_{i} \in R \quad \varepsilon_{i} \in C$

and where the normalization condition must be verified:

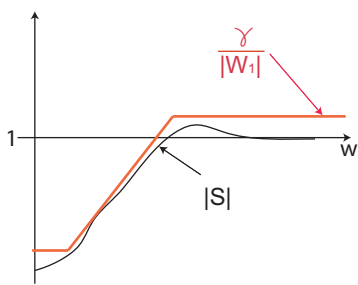

(a)

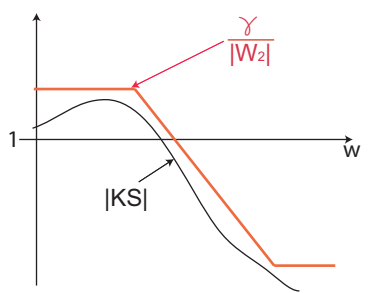

(c)

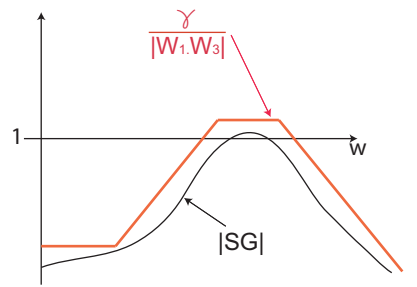

(b)

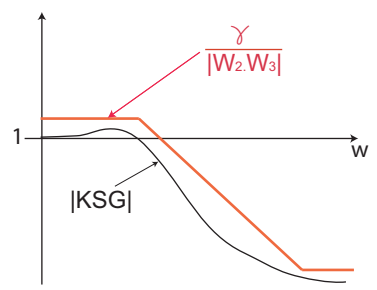

(d)
Fig. 8. Appearances of the bode diagrams of the gabarits.

$$
\|\Delta\|_{\infty}<1 \Leftrightarrow\left(\left\|\Delta_{i}(s)\right\|<1 ;-1<\partial_{i}<1 ;\left|\varepsilon_{i}\right|<1\right)
$$

Fig. 9-a represents the augmented system $P$ connected by LFT-lower with the controller $K$ and by LFT-upper with the uncertainty $\Delta$. The goal of the $\mu$-synthesis is to find the optimal controller $K$ so that $H_{\infty}$ norm of the transfer between ext - input and ext - output remains smaller than 1. According to the Small-Gain theorem [56][60], that condition is obtained if and only if the structure in Fig. 9-b remains stable whatever the fictive uncertainty $\Delta_{f}$ is, such as, $\left\|\Delta_{f}(p)\right\|_{\infty}<1$. The latter condition is equivalent to:

$$
\forall \omega \quad \mu_{\bar{\Delta}}\left(F_{l}(P(j \omega), K(j \omega))\right) \leq 1
$$

where $\bar{\Delta}$ takes into account $\Delta$ and $\Delta_{f} . F_{l}(P(j \omega), K(j \omega))$ is the connexion of $P$ on its lower part with $K$.

$\mu_{\bar{\Delta}}(M)$ represents the structured singular value of a system $M$ relatively to $\bar{\Delta} .1 / \mu_{\bar{\Delta}}(M)$ represents the minimal uncertainty $\left\{\Delta, \Delta_{f}\right\} \in \bar{\Delta}$ which destabilizes $M$.

$\mu_{\bar{\Delta}}(M)$ is defined as follow:

$\left\{\begin{aligned} & \mu_{\bar{\Delta}}(M)::=\frac{1}{\inf _{\left\{\Delta, \Delta_{f}\right\} \in \bar{\Delta}}(\bar{\sigma}(\Delta): \operatorname{det}(I-\Delta M)=0)} \\ & \mu_{\bar{\Delta}}(M):=0 \quad \text { if } \forall\left\{\Delta, \Delta_{f}\right\} \in \bar{\Delta}: \operatorname{det}(I-\Delta M) \neq 0\end{aligned}\right.$

$\bar{\sigma}(\Delta)$ is the maximal singular value of $\left\{\Delta, \Delta_{f}\right\}$.

One of the most used methods to solve the problem (15) is the $D-K$-iteration [56]. This method will be used for our application. 


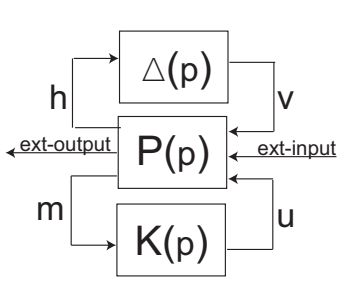

(a)

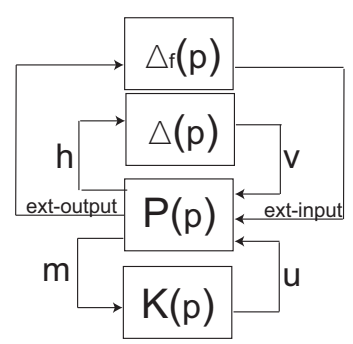

(b)
Fig. 9. a: robust synthesis. b: configuration for the $\mu$-synthesis.

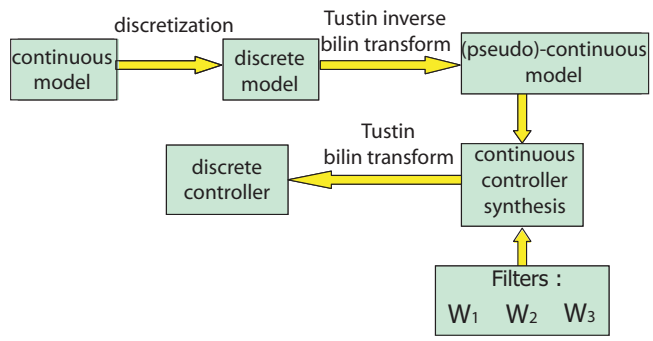

Fig. 10. The stages to synthesize a discrete controller.

As the controller is intended to be implemented in a microcontroller, this paper synthesizes a discrete controller. Nevertheless, the best and simplest way to design a discrete $H_{\infty}$ based optimal controller is via bilinear transformation [59][60][61], ie, working in an equivalent continuous domain of the discrete plant. The main reasons are that the continuous analysis is simplest, more standard and possesses more physical sense than the discrete analysis (Fig. 10). The discretization of the continous model (first step of the transformation in the figure) is necessary in order to take into account the sampling time on the model.

The bilinear transformation is defined as a bijective application $p=f(z)$, where $p$ indicates the Laplace variable and $z$ the discrete operator:

$$
p=\frac{\chi_{1} \cdot z+\chi_{2}}{\chi_{3} \cdot z+\chi_{4}}
$$

In Tustin bilinear transformation, we have $\chi_{1}=-\chi_{2}=2$ and $\chi_{3}=\chi_{4}=T_{e}$ where $T_{e}$ is the sampling time. From a discrete system, the inverse Tustin bilinear transformation gives a continuous model where the stability analysis may be done.

\section{B. Experimental application}

The experimental setup is shown in Fig. 11-a. An optical sensor with a resolution of $10 \mathrm{~nm}$ and an accuracy of $40 \mathrm{~nm}$

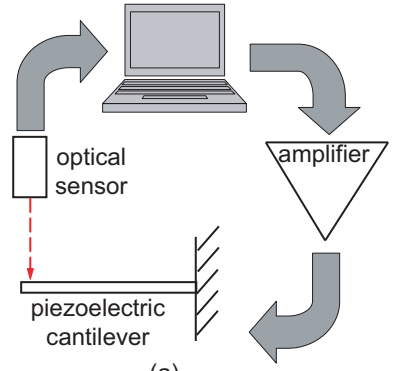

(a)

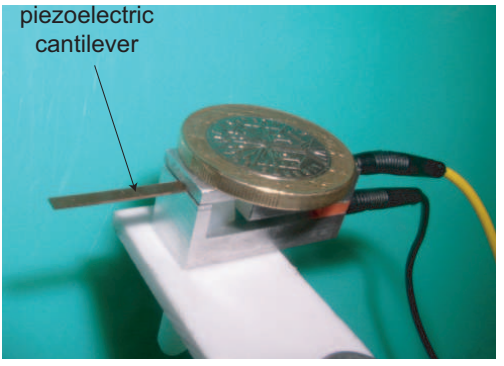

(b)

Fig. 11. a : the experimental setup principle. $b$ : photography of the cantilever.

is used. We use a dSpace real-time calculator, a computer and the Matlab-Simulink software to control the process.

The characteristics of the choosen piezoelectric cantilever give the required deflexion with low voltage $(<100 \mathrm{~V})$. Thereby, the gabarit $\gamma / W_{2}$ will not be considered. The initial continuous model (before discretization) is based on the nominal model in (9). From the (9) and (5), the uncertainty $\Delta=\{r:-1<r<1\}$ is built (see Fig. 12).

Among the specifications, the response time must be less than $10 \mathrm{~ms}$, the statical error less than $0.1 \%$ and the statical error due to perturbation less than $\frac{1}{3} \mu m / m N$.So, we propose the following gabarits:

$$
\left\{\begin{array}{l}
\frac{1}{W_{1}}=10^{-3} \times \frac{(3 p+1)}{(0.003 p+1)} \\
\frac{1}{W_{1} W_{3}}=10^{-7} \times \frac{(0.02 p+1)}{(0.0002 p+1)}
\end{array}\right.
$$

The sampling time is $T_{e}=0.2 \mathrm{~ms}$. The obtained controller $K$ has a $9^{\text {th }}$ order structure. We reduced the order to 7 by using the equilibrium reduction:

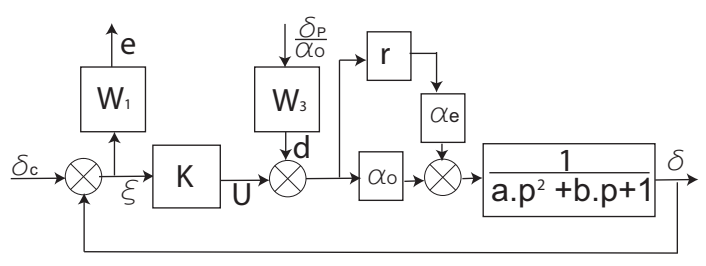

Fig. 12. Bloc-scheme including the uncertainty. 

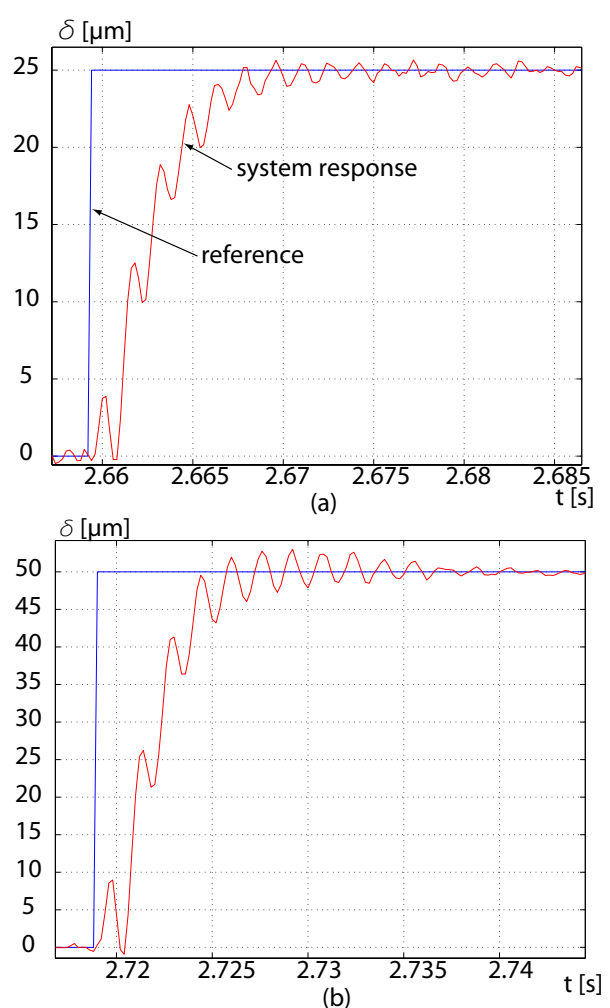

Fig. 13. Results with $\mu$-synthesis control. a: application of a serie of steps. b: zoom in a step.

$$
\left\{\begin{array}{c}
K=\frac{\begin{array}{l}
7.127 \times 10^{5} \mathrm{z}^{7}-3.232 \times 10^{6} \mathrm{z}^{6}+5.551 \times 10^{6} \mathrm{z}^{5} \\
-3.265 \times 10^{6} \mathrm{z}^{4}-2.629 \times 10^{6} \mathrm{z}^{3}+5.619 \times 10^{6} \mathrm{z}^{2}
\end{array}}{-3.634 \times 10^{6} \mathrm{z}+8.775 \times 10^{5}} \\
\mathrm{z}^{7}-3.234 \mathrm{z}^{6}+3.185 \mathrm{z}^{5}+0.7548 \mathrm{z}^{4} \\
-3.786 \mathrm{z}^{3}+2.571 \mathrm{z}^{2}-0.3252 \mathrm{z}-0.166
\end{array}\right.
$$

The experiments give good results. The Fig. 13 show the responses obtained with $25 \mu \mathrm{m}$ and $50 \mu \mathrm{m}$ of steps input signal (reference). The response time is around $8 \mathrm{~ms}$ and the statical error corresponds to the specifications. The control voltages never exceed $100 \mathrm{~V}$ (Fig. 14).

\section{CONCLUSION}

In this paper,we first present a survey on the modeling and the control of bending piezoelectric actuators notably in the case where the nonlinearities (hysteresis and creeping) are taken into account. After that, we propose a method to model these nonlinearities using the plurilinear principle. One of the main advantages of this principle is that it requires less realtime resources (memory and calculus) than other methods. In particular if the actuator is used in the non-saturated hysteresis domain, the quadrilateral modeling is a good

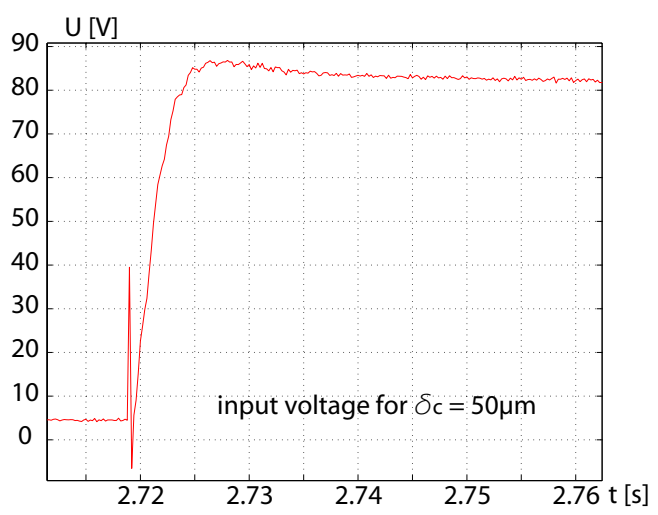

Fig. 14. Input voltage obtained with a setpoint of $50 \mu \mathrm{m}$.

approximation. In the other hand, the creep phenomenon is taken into account in the model. Knowing the uncertainty, a discrete $\mu$-synthesis robust controller is presented in order to maintain performances required in micromanipulation. It has been demonstrated that the resulting controller gives good performances. The next stage will be the study of the force control.

\section{REFERENCES}

[1] D.L. Heiserman, 'Piezoelectric polymer micromanipulator', United States Patent, $\mathrm{N}^{\circ}$ US 4,610,475, 1986.

[2] Y. Haddab, N. Chaillet and A. Bourjault, 'A microgripper using smart piezoelectric actuators', Proc. of IEEE/RSJ International Conference on Intelligent Robot and Systems, Takamatsu - Japon, 2000.

[3] J. Agnus, J.M. Breguet, N. Chaillet, O. Cois, P. de Lit, A. Ferreira, P. Melchior, C. Pellet and J. Sabatier, 'A smart microrobot on chip: design, identification and modeling', Proc. IEEE/ASME International Conference on Advanced Intelligent Mechatronics - AIM'2003, Kobe Japan, pp.685-690.

[4] E. Descouvières, D. Gendreau, P. Lutz and F. Kieffer, 'Specifications of technical information system dedicated to a re-organizable and reconfigurable microfactory', 4th International Workshop on Microfactory IWMF, oct. 2004, Shangaï China.

[5] M. Rakotondrabe, Y. Haddab, P. Lutz, "Modular and re-organizable micromanipulation station" Proceedings of the Workshop on Microrobotics, Journées du RTP-Microrobotique, December 2004, Lausanne, Switzerland.

[6] IEEE Standard on Piezoelectricity. Institute of Electrical and Electronics Engineers. Std 176-1978, september 1978.

[7] J.G. Smits and W.S. Choi, 'The constituent equations of piezoelectric heterogeneous bimorphs', IEEE Transactions on Ultrasonics, Ferroelectrics and Frequency Control, Vol.38, N`38, May 1991.

[8] J.G. Smits and W.S. Choi, 'Equations of state including the thermal domain of piezoelectric and pyroelctric heterogeneous bimorph', Ferroelectrics, Vol.141, pp271-276, 1993.

[9] N.N. Rogacheva, C.C. Chou and S.H. Chang, 'Electromechanical analysis of a symmetric piezoelectrical/elastic laminate structure:theory and experiment', IEEE Transactions on Ultrasonics, Ferroelectrics and Frequency Control, Vol.45, N2, March 1998.

[10] S.H. Chang and C.C. Chou, 'Electromechanical analysis of an asymmetric piezoelectrical/elastic laminate structure:theory and experiment', IEEE Transactions on Ultrasonics, Ferroelectrics and Frequency Control, Vol.46, $\mathrm{N}^{\circ} 2$, March 1999.

[11] M.S. Weinberg, 'Working equations for piezoelectric actuators and sensors', IEEE ASME, Journal of MEMS, Vol.8, N 4, 1999.

[12] M. Omura, H. Adachi and Y. Ishibash, 'Simulations of Ferroelectric Characteristics Using a One-Dimensional Lattice Model', Japanese Journal of Applied Physics, 30 (1991), pp. 2384-2387. 
[13] Ralph C. Smith and Zoubeida Ounaies, 'A hysteresis model for piezoceramic materials', NASA-ICASE Report N99-229 August 1999.

[14] Ralph C. Smith, Zoubeida Ounaies and Robert Wieman, 'A model for rate-dependent hysteresis inpiezoceramic materials operating at low frequencies', NASA-ICASE Report $\mathrm{N}^{\circ}$ 2001-26 September 2001.

[15] X.D. Zhang and C.A Rogers, 'A macroscopic phenomenological formulation for coupled electromechanical effects in piezoelectricity', Journal of Intelligent material systems and structures, 4 (1993), pp307316.

[16] Ping Ge and Musa Jouaneh, 'Modeling hysteresis in piezoelectric actuators', Precision engineering. 17,pp 211-221 (1995).

[17] M. Goldfarb and N. Celanovic, 'A lumped parameter electromechanical model for describing the nonlinear behavior of piezoelectric actuators', Journal of dynamc systems, measurement and control, 119:478-485, 1997.

[18] Ping Ge and Musa Jouaneh, 'Generalised preisach model for hysteresis nonlinearity of piezoelectric actuators', Precision Engineering, 20, 99111 (1997).

[19] W.S. Galinaittis and R.C. Rogers, 'Compensation for hysteresis using bivariate preisach models', SPIE smart structures and materials, mathematics and control in smart structures, San Diego, CA, 1997.

[20] G. Robert, D. Damjanovic and N. Setter, 'Preisach distribution function approach to piezoelectric nonlinearity and hysteresis', Journal of Applied Physics - September, 2001 - Volume 90, Issue 5, pp. 24592464.

[21] R.B. Mrad and H. Hu, 'A model for voltage to displacement dynamics in piezoceramic actuators subject to dynamic-voltage excitations', IEEE Transactions on mechatronics 7(4), 479-489 2002.

[22] Alfredo Dubra, John S. Massa and Carl Paterson, 'Preisach classical and nonlinear modeling of hysteresis in piezoceramis deformable mirror', OPTICS EXPRESS, Oct. 2005 Vol.13 N²2.

[23] Sun Lining, Ru Changhai, Rong Weibin, Chen Liguo and Kong Minxiu, 'Tracking control of piezoelectric actuator based on a new mathematical model', 2004 Journal in Micromech. Microeng. 14 pp14391444, August 2004.

[24] T.S. Low and W. Guo, 'Modeling of a three-layer piezoelectric bimorph beam with hysteresis', J. Microelectromech. Syst., vol.4, pp230-237, 1995.

[25] H. Jung, J.Y. Shim and D. Gweon, 'New open-loop actuating method of piezoelectric actuators for removing hysteresis and creep', REVIEW OF SCIENTIFIC INSTRUMENTS 71 (9): 3436-3440 SEP 2000.

[26] K. Kuhnen and H. Janocha, 'Modeling of transfer characteristic of piezoelectric transducers by creep and hysteresis operators'.

[27] D. Croft, G. Shed and S. Devasia, 'Creep, hysteresis and vibration compensation for piezoactuators:atomic force microscopy application', Journal of dynamic systems, measurement and control, ASME, vol.123, pp35-43, 2001.

[28] M. Goldfarb and N. Celanovic, 'Modeling piezoelectric stack actuators for control of micromanipulation', IEEE Control Systems Magazine, , Jun 1997, vol.17, pp69-79.

[29] H. Hu and Ben Mrad, 'A discrete-time compensation algorithm for hysteresis in piezoceramic actuators', Mechanical systems and signal processing 18, 169-185 (2003).

[30] K. Kuhnen and H. Janocha, 'Compensation of the creep and hysteresis effects of piezoelectric actuators with inverse systems'.

[31] Hewon Jung, Jong Youp Shim and DaeGab Gweon, 'Tracking control of piezoelectric actuators' 2001 Nanotechnology 12 14-20.

[32] Juan Manuel Cruz-Hernandez and Vincent Hayward, 'On the linear compensation of hysteresis' 36th IEEE Conference on Decision and Control, vol.1, pp1956-57, 1997.

[33] Juan Manuel Cruz-Hernandez and Vincent Hayward, 'An approach to reduction of hysteresis in smart materials' Proc. IEEE Int. on Robotic and Automation, Leuven, Belgium, pp.1510-1515, 1998.

[34] R.H. Comstock, 'Charge control of piezoelectric actuators to reduce hysteresis effects' United States Patent, brevet ${ }^{\circ}$ US 4,263,527, 1981.

[35] J.A. Main, E. Garcia and D.V. Newton, 'Precision position control of piezoelectric actuators using charge feedback' Journal of guidance, Control and Dynamics, 18(5):1068-1073, 1995.

[36] C.V. Newcomb and I. Flinn, 'Improving the linearity of piezoelectric actuators' Journal of electronics letters, 18(11):442-443, 1982.
[37] D. Newton, J. Main and E. Garcia, 'Piezoelectric actuation systems: optimisation of driving electronics' Proceedings of SPIE, vol.2717 pp.259-266, 1996.

[38] H. Janocha, D. Jendritza and P. Scheer, 'Smart actuators with piezoelectric materials' Proc of third ICIM/ECSSM, Lyon - France, pp 603609.

[39] Joël Agnus, 'Contribution à la micromanipulation: étude, réalisation, caractérisation et commande d'une micropince pézoélectrique' $\mathrm{PhD}$ Thesis LAB - CNRS, Université de Franche-Comté (France), Nov 2003.

[40] J. Agnus and N. Chaillet, 'Dispositif de commande d'un actionneur piézoélectrique et scanner muni de ceux-ci' INPI, patent, $\mathrm{N}^{\circ} \mathrm{FR} 03$ 000532, 2003.

[41] S B Choi, S S Han and Y S Lee, 'Fine motion control of a moving stage using a piezoactuator associated with a displacement amplifier' Smart Mater. Struct. 14 222-230, Dec.2004.

[42] H. Janocha, 'Adaptronics and smart structures', Chapter Actuators in Adaptronics, Springer Verlag, 1999.

[43] P. Ge and M. Jouaneh , 'Tracking control of a piezoceramic actuator', IEEE Transactions on Control System Technology, 4, pp.209-216, 1996.

[44] Ben M. Chen, Tong H. Lee, Chang-Chieh Hang, Yi Guo and Siri Weerasooriya, 'An Hinfini almost disturbance decoupling robust controller design for a piezoelectric bimorph actuator with hysteresis', IEEE Transactions on control systems technology, vol.7, $\mathrm{N}^{\circ} 2$, Mar 1999.

[45] Jinghua Zhong, Stefan Seelecke, Ralph C. Smith and Christof Büskens, 'Optimal control of piezoceramic actuators'.

[46] Robert B. Gorbet, Kirsten A. Morris and David W.L. Wang, 'Passivitybased stability and control of hysteresis in smart actuators', IEEE Transactions on control systems technology, vol.9, $\mathrm{N}^{\circ} 1$, January 2001.

[47] C.J. Li and S.Y. Li, 'On-line roundness error compensation via Pintegrator learning control', Transactions of the ASME, vol.114, pp476480, nov 1992.

[48] Jianming Yu and C. James Li, 'Robust control of piezo actuator for active error compensation in diamond turning', Transactions of the ASME, vol.114, pp476-480, nov 1992.

[49] J.D. Rasmussen, T.C. Tsao, R.D. Hanson and S.G. Kapoor, 'Dynamic variable depth cut matchining using piezoelectric actuators', Int. J. Mach Tools Manufact., vol.34, $\mathrm{N}^{\circ} 3$, pp379-392, 1994.

[50] S. Cetinkunt and A. Donmez, 'CCMAC learning controller for servo control of high precision machine tool', TP69-16:40 Proc of the American Control Conference, San Francisco, California, june 1993.

[51] U. Pinsopon and S. Cetinkunt, 'Design, fabrication and real-time neural network control of a piezo-electric actuated toolpost', DSC-vol.57-2, 1995 IMECE, Proc. Of the ASME Dynamic Systems and Control Division.

[52] J.H. Xu, 'Neural network control of a piezo tool positioner', 1993 Canadian Conf. on Electrical Computer Engr., Vancouver, BC.CAN.

[53] Dongwoo Song and C. James LI, 'Piezo actuator hybrid modelling for nonlinear inverse control in diamond turning'.

[54] K. Glover and J.C. Doyle, 'State-space formulae for all stabilizing controllers that satisfy an $H_{\infty}$-norm bound and relations to risk sensivity', Systems \& Control Letters, vol.11, pp.167-172, 1988.

[55] J.C. Doyle, K. Glover, P.K. Khargonekar and B.A. Francis, 'Statespace solutions to standard $H_{2}$ and $H_{\infty}$ control problems', IEEE Trans. Autom. Control, AC $34 \mathrm{~N}^{\circ} 8$, pp.831-846, 1989.

[56] J.C. Doyle, 'Structured uncertainty in control systems design', 24th Conf on Decision and Control, pp.260-265, Ft Lauderdale, Floride, 1985.

[57] G. Stein and J. Doyle, 'Beyond singular values and loopshapes', AIAA Journal of Guidance and Control, vol. 14, num. 1, pp. 5-16, January, 1991.

[58] G.J. Balas, J.C. Doyle, K. Glover, A. Packard and R. Smith, ' $\mu-$ analysis and synthesis toolbox for use with Matlab', Matlab-User's Guide, version-3.

[59] T. Chen and B.A. Francis, 'Optimal sampled-data control systems', Springer-Verlag, London.

[60] K. Zhou, J.C. Doyle and K. Glover, 'Robust and optimal control', Prentice-Hall, Englewood Cliffs, NJ.

[61] Leonid Mirkin, 'On discrete-time $H^{\infty}$ problem with a strictly proper controller', International Journal of Control 66, $\mathrm{N}^{\circ} 5$, pp.747-765, 1997. 